\title{
Editorial
}

\section{Dienstleistungen im Strukturwandel}

Behalten die Theoretiker des Strukturwandels C. Clark (1957), J. Fourastié (1954) und D. Bell (1975) Recht? Ist die „Dienstleistungsgesellschaft“ die „große Hoffnung“ des ausgehenden zwanzigsten Jahrhunderts in bezug auf die Wiederherstellung eines Arbeitsmarktgleichgewichts und die Verbesserung der Arbeitsbedingungen? Gemäß Fourastiés „Drei-Sektoren-Hypothese“ wird sich der Anteil des funktional, d. h. unabhängig von institutionellen Zuordnungen definierten Dienstleistungssektors sowohl an der Produktion als auch an der Beschäftigung auf achtzig Prozent erhöhen.

Entspricht die österreichische Entwicklung diesem behaupteten Trend? Nominell stieg der Anteil des institutionell abgegrenzten Dienstleistungssektors am Bruttoinlandsprodukt von $44,4 \%$ im Jahre 1964 auf $56,3 \%$ im Jahre 1984 . Im selben Zeitraum nahm der Anteil des tertiären Sektors an der Gesamtzahl der Erwerbstätigen von $39,5 \%$ auf $54,7 \%$ zu. Berücksichtigt werden muß in diesem Zusammenhang aber auch die „Tertiärisierung der Sachgüterproduktion": innerhalb des sekundären Sektors nahm die relative Bedeutung von Erwerbstätigen mit Dienstleistungsfunktionen zu.

Ein erster Blick auf die Statistik bestätigt somit den „Trend zur Dienstleistungsgesellschaft". Lassen sich daraus die Tendenzen der Zukunft und berechtigte Hoffnungen auf die Wiederherstellung eines Arbeitsmarktgleichgewichts ableiten? Werden die Beschäftigungsverluste im primären und sekundären Sektor dank eines „unstillbaren individuellen und kollektiven Hungers nach Tertiärem" mehr als kompensiert? Gegen diese Erwartung wurden gewichtige Einwände vorgebracht.

Die Theoretiker der Dienstleistungsgesellschaft stützten ihre Überlegungen auf zwei Hypothesen: 1 . Bei steigendem durchschnittlichen Pro-Kopf-Realeinkommen nimmt die reale Pro-Kopf-Nachfrage nach Dienstleistungen überproportional zu. 2. Die durchschnittliche Wachstumsrate der Arbeitsproduktivität ist im Dienstleistungssektor deutlich geringer als im Sektor Sachgüterproduktion.

Mehrere Einwände beziehen sich auf die erstgenannte Hypothese. Die Einkommenselastizität der Nachfrage ist erstens für die einzelnen Bereiche des Dienstleistungssektors 
stark unterschiedlich; keinesfalls ist für alle die postulierte hohe Elastizität feststellbar.

Trotz der geringen Produktivitätszunahme im Dienstleistungssektor entspricht die durchschnittliche Zuwachsrate von Löhnen und Gehältern in diesem Bereich jener in der Sachgüterproduktion. Produktionskosten und Preise nehmen daher im Dienstleistungssektor rascher zu. Dies hat im Drei-Sektoren-Modell jedoch keine Auswirkungen auf die Nachfrage der Dienstleistungen: implizit wird eine Preiselastizität unterstellt, die deutlich geringer als die Einkommenselastizität ist. Der zweite Einwand richtet sich gegen diese Annahme. Jedenfalls dort, wo der Verbraucher auf Substitute ausweichen kann, reagiert die Dienstleistungsnachfrage preiselastisch. Die industrielle Massenproduktion von langlebigen Konsumgütern, die einfach zu bedienen sind, schuf die Grundlage für die „Industrialisierung“ der Haushalte. Die zunehmende relative Verteuerung vieler kommerzieller Dienstleistungen und die steigende Produktivität der „Selbstbedienung“ der privaten Haushalte veranlassen letztere zur Substitution. In erster Linie sind es die der Rationalisierung kaum zugänglichen personengebundenen und sachbezogenen Dienstleistungen, die tendenziell durch Industrieprodukte, massenhaft vervielfältigte Software und die Ausweitung der Selbstbedienung vom Markt verdrängt werden. Dieser Trend zur „Selbstbedienungsgesellschaft" (J. Gershuny 1978) stellt zumindest eine Verlangsamung des Übergangs zur „Dienstleistungsgesellschaft“ dar.

Zwei Faktoren können - wie von J. Skolka (1976) erstmals dargelegt - diesen Substitutionsproze $\beta$ beschleunigen oder bremsen: die Struktur und das Ausmaß der sektoralen Lohnunterschiede sowie die Abgabenbelastung des Faktors Arbeit. Das Ausmaß der effektiven Nachfrage nach vielen Dienstleistungen hängt demnach eher von der Struktur der Einkommensunterschiede als vom Niveau der Durchschnittseinkommen ab - dies ist der dritte Einwand. Die Abgabenbelastung von Dienstleistungen beeinflußt die Produktivitätsschwelle, welche kommerzielle Dienstleistungen erreichen müssen, um auf dem Markt nachgefragt zu werden. Bei hoher Lohndifferenzierung und niedriger Abgabenquote können sich die genannten privaten Dienstleistungen trotz niedriger Produktivität noch länger auf dem Markt behaupten. Die außergewöhnliche Expansion des Dienstleistungssektors in den Vereinigten Staaten ist zum Teil auf eine derartige Konstellation zurückzuführen.

Die Wirkung der im Durchschnitt über eins liegenden Einkommenselastizität der Nachfrage nach Dienstleistungen wird somit durch die negative Preiselastizität aufgewogen. Der reale Anteil der dienstleistungsintensiven Aufwandsar- 
ten am privaten Verbrauch bleibt dann fast konstant, der nominelle Anteil steigt. Tatsächlich veränderte sich die nominelle Struktur der Konsumausgaben in Österreich wesentlich: (vgl. Skolka 1986), die Quote der dienstleistungsintensiven Aufwandsarten nahm von 32,5\% (1964) auf 50,1\% (1984) zu. Die realen Verschiebungen fielen weit schwächer aus: der Anteil der Ausgaben für die dienstleistungsintensiven Aufwandsarten erhöhte sich (zu Preisen von 1976) von $39,8 \%$ (1964) auf 44,0\% (1976), und stieg dann nur noch geringfügig auf $45,8 \%$ (1984). Auch in bezug auf die Produktionsstruktur ist diese deutliche Diskrepanz zwischen nominellen und realen Veränderungen feststellbar. Zu Preisen von 1976 nahm der Anteil des institutionell abgegrenzten Dienstleistungssektors an der Gesamtproduktion nur leicht zu, nämlich von $51,6 \%$ (1964) auf 53,6\% (1984).

Ein viertes Argument wendet sich gegen die Hypothese, wonach der private Verbrauch unmittelbar ausschlaggebend für die gesamte Dienstleistungsnachfrage sei. Dabei wird zum einen darauf verwiesen, da $ß$ in der jüngeren Vergangenheit die stärksten Wachstumsimpulse für den Dienstleistungssektor von der Nachfrage der Güterproduzenten nach bestimmten Dienstleistungen ausgingen. $\mathrm{Zu}$ diesen produktionsbezogenen Dienstleistungen, die als Zwischenprodukte in die Herstellung von Industriegütern eingehen, zählen Forschung und Entwicklung, Finanzierung, Versicherung, Marktforschung, Organisation, Arbeitsvorbereitung, Rechnungswesen, Werbung, Transport usw. Die überproportionale Zunahme produktionsbezogener Dienstleistungen impliziert erstens, daß die Bedeutung des privaten Dienstleistungsverbrauchs und der entsprechenden Präferenzen für die Entwicklung des Dienstleistungssektors rückläufig sind. Zweitens wird die zunehmende Interdependenz zwischen sekundärem und tertiärem Sektor evident.

Zum anderen widerlegt auch die kräftige Expansion der öffentlichen Dienste die Nachfragehypothese des Drei-Sektoren-Modells. Da die öffentliche Hand nicht nur Dienstleistungen für Endverbraucher zur Verfügung stellt und die Präferenzen der Konsumenten nur mittelbar, nämlich über einen politischen Proze $\beta$, vermittelt werden, kann auch kein unmittelbarer Wirkungszusammenhang zwischen der Präferenzhierarchie und der Einkommensentwicklung der privaten Haushalte einerseits und der Ausweitung der Dienstleistungen im öffentlichen Bereich andererseits hergestellt werden.

Fünftens ist die Zunahme der Arbeitsproduktivität in den einzelnen Bereichen des Dienstleistungssektors stark unterschiedlich und keineswegs generell geringer als in der Sachgüterproduktion. J. W. Baumol (1985) differenziert je nach 
Verlauf des Produktivitätstrends drei Kategorien der Dienstleistungen:

- „Progressive“ Dienstleistungen: Hier besteht kaum direkter Kontakt zwischen Produzenten und Konsumenten. Technische Innovationen haben daher einen außergewöhnlichen Produktivitätsfortschritt ermöglicht, wie am Beispiel der revolutionären Entwicklung der Telekommunikation zu sehen ist.

- „Asymptotisch stagnierende“ Dienstleistungen: Der Lebenszyklus dieser Dienstleistungen umfaßt Phasen mit raschem Produktivitätsanstieg und ausgeprägten Kostensenkungen. Da sich der rasante Produktivitätsfortschritt nur auf einen Teil der erforderlichen Inputs (z. B. Computer-Hardware) beschränkt, nimmt die Wachstumsrate der Arbeitsproduktivität fortlaufend ab. Der Kostenanteil der der Rationalisierung zugänglichen Hardware-Produktion geht rasch zurück, während der entsprechende Anteil der Software-Herstellung, die dem „stagnierenden“ Dienstleistungstyp (siehe unten) zuzuordnen ist, ständig steigt. Im Falle der Rundfunk- und Fernsehübertragung sind die entsprechenden Input-Bestandteile die Übertragungstechnik (,progressiv“) bzw. die Produktion der Programme (,stagnierend“). Die Produktivitäts- und Kostentrends nähern sich daher asymptotisch den im "stagnierenden“ Bereich vorherrschenden Tendenzen, sofern die Möglichkeiten der Substitution von Arbeit durch Kapital begrenzt sind.

- „Stagnierende“ Dienstleistungen setzten meistens den direkten Kontakt zwischen Produzenten und Konsumenten voraus. Ihre Qualität hängt hauptsächlich von der aufgewandten Zeit oder sogar von der aktiven Mitwirkung der Klienten ab (z. B. Lehrer, Sozialhelfer, Psychotherapeuten). Der zeitliche Verlauf der Nachfrage ist meist unregelmäßig; da nicht auf Lager produziert werden kann, schwanken Arbeits- und Kapitalauslastung. Aufgrund der genannten Charakteristika stagniert die Produktivität oder steigt nur geringfügig.

Dies hatte, worauf bereits verwiesen wurde, die Substitution verschiedener persönlicher Dienstleistungen durch Haushaltsgeräte und Selbstbedienung oder die Übernahme der Leistungserstellung durch die öffentliche Hand zur Folge. Auch bei vielen „stagnierenden“ Dienstleistungen sind jedoch Produktivitätsfortschritte zu erzielen, falls einzelne Inputs, welche für die Produktion personenbezogener Dienstleistungen herangezogen werden, der Rationalisierung zugänglich sind und/oder grundlegende technische und organisatorische Innovationen erfolgen. Letzteres wird am Beispiel der Informations- und Unterhaltungsdienste 
deutlich. Durch die Zwischenschaltung eines Speichermediums wird die gleichzeitige Anwesenheit von Produzenten und Konsumenten am selben Ort überflüssig. Dies erlaubt das Ausschöpfen zeitlicher Einsparungsmöglichkeiten in der Produktion. Vor allem aber die massenhafte Vervielfältigung auf Speichermedien führt zu einer wesentlichen Verminderung der Stückkosten. Ein einmaliger Produktivitätsdurchbruch verwandelt mithin manche ,stagnierende“ Dienstleistung in eine solche des Typs „asymptotisch stagnierend".

Über die zukünftige Entwicklung des intersektoralen Produktivitätsunterschieds gehen die Meinungen weit auseinander. Die IAB-Prognos-Studie für die BRD rechnet (in der mittleren Variante, d. h. bei einer durchschnittlichen jährlichen Wachstumsrate des realen BIP von 2,5\% zwischen 1982 und 2000) damit, daß die Produktivität in den produktionsbezogenen Dienstleistungen (siehe oben) etwas rascher zunehmen wird als in der Sachgüterproduktion und der relative Rückstand insbesondere des Handels, aber auch der öffentlichen Dienste abnehmen wird. Insgesamt würde sich daher das intersektorale Produktivitätsgefälle deutlich vermindern. Alle Studien, welche die relative Produktivitätsentwicklung im Dienstleistungssektor optimistisch einschätzen, begründen dies vor allem mit unausgeschöpftem Potential zur Erhöhung der Kapitalintensität sowie zunehmender Arbeitsteilung.

Weiters führen Kritiker der „Drei-Sektoren-Hypothese“ an, daß diese für eine geschlossene Wirtschaft formuliert worden sei. Bei der Anwendung auf offene Wirtschaften verliert das Modell stark an Aussagekraft.

Letztlich wird gegen die „Drei-Sektoren-Hypothese“ vorgebracht, daß die ihr zugrundeliegende Gliederung der Gesamtwirtschaft für viele Fragestellungen, so auch im Zusammenhang mit der Beschäftigungs- und Strukturpolitik, nicht sinnvoll sei. Dieser Standpunkt läßt sich auch aus den bisher präsentierten Argumenten ableiten. Erstens ist der Dienstleistungssektor, gleichgültig, ob funktional oder institutionell definiert, in nahezu jeder Hinsicht äußerst heterogen. Verallgemeinernde Thesen sind daher zumindest problematisch, wenn nicht sogar irreführend. Zweitens ist die Trennung zwischen Sachgüterproduktion und Dienstleistungssektor für analytische Zwecke gerade im Hinblick auf Beschäftigungs- und Strukturpolitik nicht zielführend. Der Dienstleistungssektor insgesamt ist kein von der Sachgüterproduktion unabhängiger Wirtschaftsbereich mit eigenständigem Entwicklungspotential, wie die „Drei-Sektoren-Hypothese“ suggeriert.

Welche wirtschaftspolitischen Schlußfolgerungen ergeben 
sich aus den bisherigen Überlegungen? $\mathrm{Ob}$ und welche Maßnahmen zugunsten von Dienstleistungsaktivitäten sinnvoll sind, kann nur für Teilbereiche diskutiert werden, wobei jeweils neben den leistungsspezifischen Besonderheiten den Beziehungen zu anderen Wirtschaftsbereichen spezielle Aufmerksamkeit zu schenken ist. Eine „Dienstleistungspolitik“ schlechthin existiert nicht.

Der Bereich der produktionsbezogenen Dienstleistungen, auf die bereits verwiesen wurde, und die Sachgüterproduktion stehen in enger wechselseitiger Abhängigkeit. Die Struktur der für die Herstellung von Gütern benötigten Tätigkeiten verändert sich zu Lasten der produzierenden und zugunsten von dienstleistenden Aktivitäten. Dies ist sowohl auf den technischen und organisatorischen Fortschritt als auch auf die zunehmende internationale Arbeitsteilung zurückzuführen.

In den westlichen Industriestaaten verliert der Anteil der in der standardisierten Massenproduktion beschäftigten Arbeitnehmer laufend an Bedeutung. Der Strukturwandel innerhalb des Sektors Sachgüterproduktion vollzieht sich in Richtung auf Präzisionsprodukte, Spezialfertigungen in kleiner Serie gemäß den Wünschen der Auftraggeber und Produkte auf der Basis fortgeschrittener Technologien (R. Reich 1983). Gegenüber der standardisierten Massenfertigung resultiert ein höherer Bedarf an produktionsbezogenen Dienstleistungen je Einheit, u. a. an Forschung und Entwicklung, Design, Weiterbildung der Mitarbeiter, Kundenberatung und -service, Werbung, Reparatur und Präzisionsprüfung, Finanzierung und Versicherung, Rechtsberatung, Reisen und Übersetzungen, Fernsprech- und Telekommunikationsdiensten, Verwaltung, Unternehmensberatung, Marktbeobachtung.

Viele der genannten Dienstleistungen tragen den Folgen der fortschreitenden Internationalisierung der Märkte - rasch wechselnde Nachfragebedingungen, zunehmende Unsicherheiten und gestiegener Bedarf an Informationsvermittlung Rechnung.

Produktionsbezogene Dienstleistungen werden zu einem integrierten Bestandteil des Warenangebots von güterproduzierenden Betrieben. Güter und ergänzende Dienstleistungen bilden ein Paket. Neben die Preis- und Qualitätskonkurrenz tritt die Konkurrenz mit zusätzlichen Dienstleistungen. Für die Wettbewerbsfähigkeit auf den Weltmärkten wird diese Kombination von wachsender Bedeutung sein.

Aus der bisherigen Argumentation wird die Interdependenz zwischen Sachgüterproduktion und produktionsbezo- 
genen Dienstleistungen deutlich. Einerseits bestimmen technische und organisatorische Änderungen in der Güterproduktion sowie Entwicklungen auf den Gütermärkten weitgehend die Nachfrage nach produktionsbezogenen Dienstleistungen. Andererseits bilden letztere einen komplementären Bestandteil arbeitsteiliger Güterproduktion und daher eine zwar nicht hinreichende, aber immer stärker notwendige Voraussetzung für den Erfolg güterproduzierender Unternehmungen, wie Branchenanalysen des DIW bestätigen.

Bestehen wirtschaftspolitische Möglichkeiten, die Ausweitung des Bereichs der produktionsbezogenen Dienstleistungen, der in Österreich im Vergleich zu anderen Industriestaaten zweifellos einen Rückstand aufweist, zu beschleunigen? Ein Ansatzpunkt ergibt sich daraus, da $ß$ neue produktionsorientierte Dienstleistungen zumeist in den produzierenden Unternehmungen mit einer Begrenzung auf interne Aufgaben erstellt werden. Die mangelnde Ausgliederung derartiger Funktionen bedingt, daß Vorteile der Spezialisierung gegenwärtig nicht wahrgenommen werden. Eine vermehrte Bereitstellung von Risikokapital und andere Maßnahmen zugunsten innovativer Dienstleistungsunternehmungen können die Entwicklung eines leistungsfähigen Marktes für produktionsbezogene Dienstleistungen unterstützen bzw. den inländischen Marktanteil anheben. Für die Etablierung auf ausländischen Märkten bestehen gegenwärtig wohl bessere Chancen als dies in der Zukunft der Fall sein wird, befinden sich diese Märkte doch derzeit erst in der Entstehungsphase.

\section{III}

In bezug auf viele verbraucherbezogene Dienstleistungen konkurriert die These vom Übergang zur Dienstleistungsgesellschaft mit jener von der Tendenz zur Selbstbedienungsgesellschaft. Prinzipiell bieten sich mehrere Möglichkeiten, die Substitution von personengebundenen und sachbezogenen Dienstleistungen durch die Kombination von Industriegütern, Software und Selbstbedienung zu verlangsamen.

In den Vereinigten Staaten besteht eine starke Differenzierung der Lohnsätze und eine vergleichsweise niedrige Abgabenquote. Sowohl die Verschärfung der Segmentierung des Arbeitsmarktes auf Kosten einiger Gruppen als auch die Senkung der Abgabenquote auf ein Niveau, das die Aufrechterhaltung der Funktionen des Wohlfahrtsstaates nicht mehr gewährleistet, sind jedoch vom Standpunkt der Arbeitnehmerschaft abzulehnen.

Der in Schweden lange Zeit verfolgte Weg, bei egalitär ausgerichteter Lohnpolitik und unter Inkaufnahme einer steigenden Abgabenquote zahlreiche soziale Dienstleistun- 
gen zunehmend durch die öffentliche Hand anzubieten, stie $\beta$ zu Beginn der achtziger Jahre an finanzielle Grenzen. Da in Östereich eine wesentliche Erhöhung der Abgabenquote zur Ausweitung der öffentlichen Dienstleistungen im Sozialbereich vermutlich nicht die Zustimmung einer Mehrheit der Wähler fände, bestünde zumindest theoretisch eine Perspektive darin, das vergleichsweise hohe Transfervolumen zugunsten eines zusätzlichen öffentlichen Dienstleistungsangebots zu verringern. Ein derartiger Umbau der Ausgabenstruktur wäre zweifellos mit erheblichen Schwierigkeiten verbunden. Abgesehen davon wären auch folgende Aspekte zu berücksichtigen: Erstens hätte die Einschränkung monetärer Transfers eine gewisse Beeinträchtigung der individuellen Entscheidungsfreiheit zur Folge. Zweitens fand die Kritik an überdimensionierten bürokratischen Organisationen gerade im Bereich des Angebots personengebundener Dienstleistungen ihren Ursprung.

Erweist sich diese Einschätzung der Hindernisse für die Ausweitung der sozialen Dienstleistungen im öffentlichen Sektor als richtig, so wird die Aufgabe in Zukunft darin bestehen, die Bereitstellung dieser Dienste (Gesundheit, Fürsorge, Bildung und Erziehung, Kultur) zu gewährleisten, ohne den Staatsanteil zu erhöhen. Die Rede ist hier, und dies sei nachdrücklich betont, keinesfalls vom Abbau öffentlicher Dienstleistungen im Sozialbereich, sondern von der Ergänzung dieses Angebots durch den privaten Sektor oder bestimmte Formen der gemeinnützigen Leistungserstellung. Letzteren könnte eine wachsende Bedeutung auf diesem Gebiet zufallen, zumal in überschaubaren, dezentralisierten Bereichen den individuellen Bedürfnissen der zu betreuenden Menschen in höherem Maße entsprochen werden kann. Private Unternehmungen werden auf die steigende Nachfrage von Beziehern gehobener Einkommen nach qualitativ hochwertigen und teuren Dienstleistungen flexibel reagieren und auf diese Weise die Angebotspalette erweitern, ohne mit den anderen Anbietern in unmittelbare Konkurrenz zu treten.

Ihre regulierende Tätigkeit im Dienste der Aufrechterhaltung bestimmter Standards der Dienstleistungen wird die öffentliche Hand beibehalten.

Eine steuerpolitische Möglichkeit, die Nachfrage nach arbeitsintensiven Dienstleistungen zu unterstützen, besteht darin, den Faktor Arbeit generell oder selektiv zu entlasten und statt dessen andere Produktionsfaktoren und den Konsum stärker zu belasten. Die gegenwärtig laufende Diskussion um die Wertschöpfungsabgabe und verschiedene Formen einer Umweltabgabe befaßt sich auch mit dieser Thematik. 
Ausschlaggebend für den Netto-Beschäftigungseffekt einer zunehmenden Tendenz zur Selbstbedienungsgesellschaft sind einerseits Innovationen im Dienstleistungsbereich. In der Selbstbedienungsgesellschaft verschwinden zwar einige traditionelle personengebundene Dienstleistungen, aufgrund von Innovationen auf dem Gebiet der Informationstechnik entsteht aber Nachfrage nach neuen "SelbstbedienungsDienstleistungen“ (J. Gershuny, I. Miles 1983). Die auf der Installierung von Breitband-Kommunikationsnetzen und Heimterminals beruhenden Neuerungen erfassen $u$.a. die Bereiche Haushaltsführung, Unterhaltung (z. B. individuell abrufbare Videofilme), Bildung und Erziehung (interaktiver Fernunterricht) und Kommunikation (Fern-Einkauf, TeleKonversation). Beschäftigungseffekte resultieren sowohl im Dienstleistungssektor, insbesondere in der Herstellung von Software, als auch in der Sachgüterproduktion (Hardware und Infrastruktur). Im Zusammenhang mit der Erstellung der Infrastruktur kommt dem Staat eine wesentliche Bedeutung zu.

Die von S. B. Linder (1970) aufgestellte und auch von F. Scharpf (1985) vertretene These, die Ausdehnung derartiger konsumbezogener Dienstleistungen stoße an Grenzen, die sich aus prinzipiell limitierten persönlichen Zeitressourcen ergeben, ist entgegenzuhalten, da 3 sich die Nachfrage in Richtung auf „höherwertige“ Dienstleistungen verschiebt, wobei die Zahl der pro Zeiteinheit konsumierbaren Dienstleistungsarbeitsstunden ansteigt.

Entscheidend für die Auswirkungen zunehmender Selbstbedienung im obigen Sinn auf die Beschäftigungsentwicklung ist andererseits die Frage, ob Güter und Software, welche zur Substitution der lohnarbeitsintensiven Dienstleistungen herangezogen werden, im Inland produziert werden können oder importiert werden müssen. Möglichkeiten wirtschaftspolitischer Aktivitäten zugunsten inländischer Produktion auf diesem Gebiet wurden bereits oben im Zusammenhang mit der produktionsbezogenen Beschäftigung angerissen.

\section{V}

Zwei weitere Instrumente, denen im Zusammenhang mit der Bewältigung des Strukturwandels eine wesentliche Rolle zufällt, sind die Arbeitsmarkt- und die Bildungspolitik. Die zukünftige Bedeutung der einzelnen skizzierten Eintwicklungstendenzen innerhalb des Dienstleistungssektors kann zwar kaum präzise eingeschätzt werden, aber allein schon 
aufgrund der außer Zweifel stehenden relativen und absoluten Zunahme der Dienstleistungsfunktionen ist ein steigender Bedarf an höher qualifiziertem Personal zu erwarten. Sowohl die österreichischen Erfahrungen in der jüngeren Vergangenheit als auch ausländische Untersuchungen deuten in diese Richtung. Die im folgenden kurz präzisierten Resultate einer diesbezüglichen Projektion für die Bundesrepublik Deutschland lassen zumindest grobe Rückschlüsse auf wahrscheinliche Trends in Österreich zu.

IAB und Prognos versuchten - ausgehend von drei Szenarien des Wirtschaftswachstums, des sektoralen Strukturwandels und der Auswirkungen technischer und sozioökonomischer Veränderungen auf Tätigkeiten - mit unterschiedlichen Projektionsmethoden die Richtung und Größenordnung des Bedarfs an Arbeitskräften unterschiedlicher Qualifikation in der BRD bis zum Jahr 2000 vorauszuschätzen. Die Ergebnisse „... . legen den Schluß nahe, daß der Wandel der sektoralen und der Tätigkeitsstrukturen eine kontinuierliche Höherqualifizierung der Erwerbstätigen bedingt. Zum einen nehmen die Tätigkeiten an Gewicht zu, die relativ hohe Anteile an qualifizierten und höher qualifizierten Arbeitskräften aufweisen (Tätigkeitsstruktureffekt), zum anderen geht innerhalb dieser - wie auch der meisten anderen - Tätigkeiten die Tendenz zur Besetzung der verfügbaren Arbeitsplätze mit Personen hoher Qualifikation weiter (Tätigkeitsbesetzungseffekt)." Der Anteil sowohl der betrieblich oder schulisch ausgebildeten Fachkräfte als auch der Hochschulabsolventen wird sich wesentlich erhöhen, jener der Erwerbstätigen mit Meister-, Techniker- oder Fachschulabschluß konstant bleiben, der relative (und absolute) Bedarf an Erwerbstätigen ohne formale Ausbildung stark zurückgehen.

Die Quote der Hochschulabsolventen wird gemäß den Projektionen in den dem funktional definierten Dienstleistungssektor zugeordneten Tätigkeitsbereichen „dispositive und Managementtätigkeiten, Forschung und Entwicklung“, „Entscheidungsvorbereitung, Bürotätigkeit“ sowie „Sonstige Dienstleistungen" (Ausbildung, Gesundheits- und Sozialdienste, Rechtspflege, Bewachung u. a.) stark ansteigen. Hohe Zuwachsraten für den Anteil der betrieblich oder schulisch ausgebildeten Fachkräfte wurden für die Tätigkeitsbereiche „Lagerung, Transport, Vertrieb“, „Entscheidungsvorbereitung, Bürotätigkeit“ und „Reinigung, Hauswirtschaft, Bewirtung" ermittelt. Den Tätigkeiten im Dienstleistungssektor kommt somit ein wesentlicher Anteil an den projizierten Veränderungen in der Qualifikationsstruktur zu.

Sowohl Dienstleistungen als auch flexiblere Fertigungsverfahren verlangen Qualifikationen, welche die eigene Entscheidungs- und Anpassungsfähigkeit sowie breites Wissen 
anstelle routinisierter Arbeit nach Anweisung in den Mittelpunkt stellen. Diese groben Einschätzungen decken sich der Richtung, weniger dem Ausmaß nach mit den Thesen von D. Bell (1975) und H. Kahn (1967) über den Qualifikationsbedarf in der „postindustriellen Gesellschaft“.

Der Dienstleistungssektor ist durch starke Heterogenität gekennzeichnet. Zahlreiche Entwicklungstendenzen, deren zukünftige Bedeutung kaum einzuschätzen ist, sind derzeit im Gange. Ausgelöst werden diese von technischen, ökonomischen und sozialen Faktoren, welche in den einzelnen Bereichen auf verschiedene Art und in unterschiedlicher Intensität wirken.

Werden sich die von Fourastié formulierten ,großen Hoffnungen“ erfüllen? Mit einiger Sicherheit kann davon ausgegangen werden, daß sich die Arbeitsbedingungen der Mehrheit der Erwerbstätigen im Zuge des strukturellen Wandels zu Dienstleistungsfunktionen verbessern werden.

Weitaus skeptischer muß die Hoffnung auf die Wiederherstellung eines Beschäftigungsgleichgewichts allein aufgrund der Beschäftigungsdynamik im Dienstleistungssektor beurteilt werden. Die Arbeitskräftenachfrage des funktional definierten Dienstleistungssektors wird zwar aller Voraussicht nach weiter steigen, aber nicht in dem von der „Drei-Sektoren-Hypothese" her zu erwartenden Ausmaß und nur zum Teil aufgrund der dort unterstellten Bestimmungsgründe. Die Entwicklung der Arbeitskräftenachfrage im tertiären Sektor steht in engem Zusammenhang mit Vorgängen in anderen Wirtschaftsbereichen. Der technische Fortschritt und das Produktivitätswachstum haben sich beschleunigt, Spielräume zur Erhöhung der Kapitalintensität sind bei weitem noch nicht ausgeschöpft. Wie groß der Netto-Beschäftigungseffekt der oben skizzierten Tendenzen innerhalb des Dienstleistungssektors, insbesondere auch der Substitutionsund Innovationsprozesse sein wird, ist unbekannt.

Am Ende der Überlegungen stehen mehr Fragen als Antworten. Jedenfalls sind simple, globale Erklärungsansätze und Lösungsvorschläge unangebracht. Ausländische Modelle können nicht ohne weiteres übernommen werden. Es ist vielmehr an der Zeit, die Probleme, Chancen und Beeinflussungsmöglichkeiten einzelner Bereiche des Dienstleistungssektors zu analysieren, ohne gleichzeitig die Zusammenhänge aus den Augen zu verlieren. 


\section{Literatur}

Baumol, William J., Productivity Policy and the Service Sector, in: Inman, Robert P. (Hrsg.), Managing the Service Economy: Prospects and Problems (Cambridge 1985), S. 301-317.

Bell, Daniel, Die nachindustrielle Gesellschaft (Frankfurt/M. 1975).

Clark, Colin, The Conditions of Economic Progress (London 1957).

Fourastié, Jean, Die große Hoffnung des 20. Jahrhunderts (Köln 1954).

Gershuny, Jonathan, After Industrial Society (London 1978).

Gershuny, Jonathan/Miles, Ian, The New Service Economy (London 1983).

Kahn, Herman/Wiener, J. Anthony, Ihr werdet es erleben (Gütersloh 1967).

Lindner, Staffan B., The Harried Leisure Class (New York 1970).

Reich, Robert B., The Next American Frontier (New York 1983).

Scharpf, Fritz W., Strukturen der post-industriellen Gesellschaft, in: Wirtschaft und Gesellschaft 1/1985, S. 9-34.

Skolka, Jiri, Der Dienstleistungssektor der österreichischen Wirtschaft, in: WIFO-Monatsberichte 9/1986, S. 584-611.

Skolka, Jiri, Long Term Effects of Unbalanced Labour Productivity Growth, in: Solari and du Pasquier (Hrsg.), Private and Enlarged Consumption (North Holland 1976), S. 279-301. 